\title{
STRUCTURE ELUCIDATION OF DEGRADATION PRODUCTS OF Z- ISOMER OF LULICONAZOLE ACTIVE PHARMACEUTICAL INGREDIENT
}

\author{
Shaik John Saida ${ }^{1,2, *}$, Manikandan A ${ }^{1}$, V.V.S.R.N.Anji Karun Mutha ${ }^{2}$, \\ Muralidharan Kaliyaperumal ${ }^{2}$, Chidananda Swamy Rumalla ${ }^{2}$, \\ Ramulu Yanaka ${ }^{2}$ and S. Venkat Rao $^{3}$ \\ ${ }^{1}$ Department of Chemistry, Bharath Institute of Higher Education and Research, Selaiyur, \\ Chennai, Tamil Nadu- 600073, India \\ ${ }^{2}$ Department of Discovery Chemistry, GVK Biosciences Pvt. Ltd, IDA Mallapur, Hyderabad, \\ Telangana-500076, India \\ ${ }^{3}$ SMS Pharmaceuticals Ltd, R\&D Center, Gagillapur, Hyderabad, Telangana-500043, India \\ *E-mail: saida_sk2005@yahoo.co.in
}

\begin{abstract}
Luliconazole geometrical isomer- $Z$ impurity, which is formed during the synthesis of Luliconazole active pharmaceutical ingredient. And isomer-Z has specific control limit is around NMT 0.50\% in line with ICH guidelines. We pursued this research to understand the fate of this isomer during degradation and hence we subjected it to forced degradation and characterized the degradation products. Five degradations products were formed and named as DP-1, DP-2, DP-3, DP-4, and DP-5 were formed in acid, base and peroxide-mediated degradation. Further, no degradation was found in thermal and photolytic conditions. All degradation impurities were isolated, and their structures were elucidated by UPLC-MS, HRMS, and NMR $\left({ }^{1} \mathrm{H},{ }^{13} \mathrm{C} \& 2 \mathrm{D}\right)$. DP-1 and DP-2 were formed in acid-mediated degradation; DP-3 was generated in peroxide-mediated oxidative degradation, while DP-4 \& DP-5 originated in base degradation.

Keywords: Luliconazole Z-isomer, Luliconazole -Active Pharmaceutical Ingredient, Degradation products, NMR, HRMS.
\end{abstract}

C $\mathrm{RASA}$ ĀYAN. All rights reserved

\section{INTRODUCTION}

Luliconazole has a unique chemical structure as the imidazole moiety is incorporated into the ketene dithioacetate structure. It is a structurally related compound of Lanoconazole. Lanoconazole has 2chlorophenyl moiety in its structure whereas Luliconazole has 2, 4-dichlorophenyl in its structure. The chemical name of Luliconazole is (E)-[4-(2, 4-dichlorophenyl)-1, 3-dithiolan-2-ylidene]-1imidazolylacetonitrile, and is shown in Fig.-1. Luliconazole has been reported to have strong in vitro antifungal activity against Trichophyton, C. albicans, and A. fumigates ${ }^{2-4}$. Luliconazole $1 \%$ cream was approved in Japan in 2005 for the treatment of tinea infections, followed by approval in November 2013 by the US Food and Drug Administration for the treatment of interdigital Tineapedis. In June 2009, the $1 \%$ cream was approved for marketing in India.<smiles>N#CC(=C1SCC(c2ccc(Cl)cc2Cl)S1)n1ccnc1</smiles>

Fig.-1: Structure of Z-isomer of Luliconazole

Rasayan J. Chem., 12(2), 691-696(2019)

http://dx.doi.org/10.31788/RJC.2019.1225087 
Geometrical and optical isomerism is possible in Luliconazole. Geometrical isomerism due to the presence of a double bond and optical isomerism because of Chiral center and therefore it exhibits total 4 isomers such as RE, RZ, SE and SZ. Luliconazole RE is biologically active substance, remaining three are biologically inactive isomers.

Luliconazole RE and SE, and RZ and SZ were separated as two peaks in liquid chromatography (LC) that means E-Isomers of $(\mathrm{R}, \mathrm{S})$-Luliconazole reflecting at one retention time and rest of Z-isomers $(\mathrm{R}, \mathrm{S})$ are reflecting at different retention time. However, there are no reports on the isolation and structure elucidation of degradants from either Luliconazole or its Z-isomer.

Among Luliconazole isomers, the percentage of $\mathrm{RZ}$ isomer was found to be more along with drug substance (RE). Therefore, Z-isomer (RZ) of Luliconazole was isolated and its degradation study was conducted to characterize its degradation products. Even though Z-isomer (RZ) of Luliconazole is biologically inactive and its percentage is $>1 \%$ in the API, the degradation pattern upon long term storage of Luliconazole is essential in characterizing the adverse events. Based on this concept, we have focused on impurity degradation instead of the drug substance. The drug was synthesized in a stereo selective manner and hence RE and RZ isomer formation was $70 \%$ and $25 \%$ respectively. The other two isomers $\mathrm{SZ}$ and $\mathrm{SE}$ formation is $2 \%$ and $3 \%$ respectively. After final isolation of drug substance, RE is not less than $99 \%$, RZ is not more than (NMT) $0.5 \%$ and the other two isomers SZ, SE was identified as NMT $0.1 \%$ or not detected (ND) level. High-Performance Liquid Chromatography (HPLC) methods have been reported for validating Luliconazole bulk drug and in cream formulation ${ }^{5}$ along with its related impurities in topical formulation ${ }^{6}$.

\section{Chemicals and Reagents}

\section{EXPERIMENTAL}

Luliconazole (Z-isomer) pure compound was received from SMS Pharmaceuticals Ltd. in Hyderabad. High pure HPLC grade solvent acetonitrile from Merck, ammonium acetate and ammonium bicarbonate from Sigma-Aldrich, Dimethyl sulfoxide-d6 with TMS, methanol-d4 with Tetramethylsilane (TMS) and D2O solvents from Cambridge isotopic limited. Milli-Q grade water was used.

\section{Liquid Chromatography-Mass Spectrometry (LC-MS)}

LCMS was used to identify the new degradation impurities of Luliconazole-Z isomer, Column: X-Bridge C-18 2.5 $\mu \mathrm{m}(3.0 \times 50 \mathrm{~mm})$, mobile phase A: $5 \mathrm{mM}$ ammonium acetate in aqueous solution; mobile phase B: $100 \%$ acetonitrile with gradient elution $\%$ B: $0 / 5,3 / 100,4.5 / 100,4.7 / 5,5.5 / 5$, diluent: buffer \& acetonitrile (50:50); wavelength of detection: $200-400 \mathrm{~nm}$.

\section{Preparative HPLC}

Gilson semi Preparative HPLC, which is a Binary gradient system with Liquid handler GX-271N connected to pump modules 331\& 332, with photodiode detector. Column: Waters X-Select C18 $(150 \times$ $19 \mathrm{~mm}) 5 \mu$, mobile phase A: $0.1 \%$ formic acid in water; mobile phase B: $100 \%$ acetonitrile, and another mobile phase A: $10 \mathrm{mM}$ ammonium bicarbonate in water; mobile phase B: $100 \%$ acetonitrile. DP-1 \& DP-2 were isolated by reverse phase purification as a mixture of two peaks with formic acid buffer mobile phase A: $0.1 \%$ formic acid in water; mobile phase B: $100 \%$ acetonitrile and gradient elution method of \%B: 0/10,8.5/80,8.6/99,10.5/99,10.6/10,13/10, DP-3: was isolated by using reverse phase purification, mobile phase A: $10 \mathrm{mM}$ ammonium bicarbonate in water, mobile phase B: $100 \%$ acetonitrile and the gradient elution method \%B: 0.0/10,3.9/30,7.2/30,7.5/99,9.5/99,9.7/10,12.5/10, DP-4 \& DP-5: were isolated by using reverse phase purification as a mixture of two peaks with formic acid buffer, mobile phase A: $0.1 \%$ formic acid in water; mobile phase B:100\% acetonitrile with gradient elution \%B: 0/20,11/85,11.5/99,13.5/99,13.7/20,16/20.

\section{Super Critical Fluid Chromatography (SFC)}

Further, degradation products (DP-1 and DP-2) E \& Z isomers were separated and isolated by using,

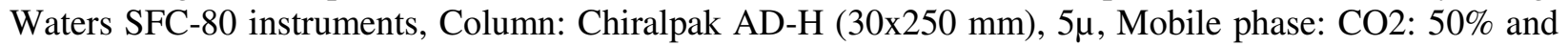
co-solvent: 50\% (ethanol). DP-4 and DP-5, E\& Z isomers were further isolated by using, Waters SFC-80 


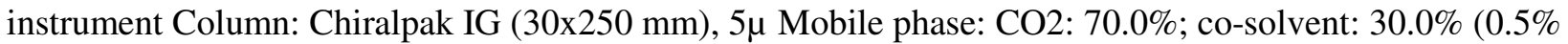
DEA in methanol); flow rate: $100.0 \mathrm{~g} / \mathrm{min}$; back pressure: 100.0 bar; UV: $215 \mathrm{~nm}$; stacked injection time: $9.2 \mathrm{~min}$; loading per injection: $4.0 \mathrm{mg}$

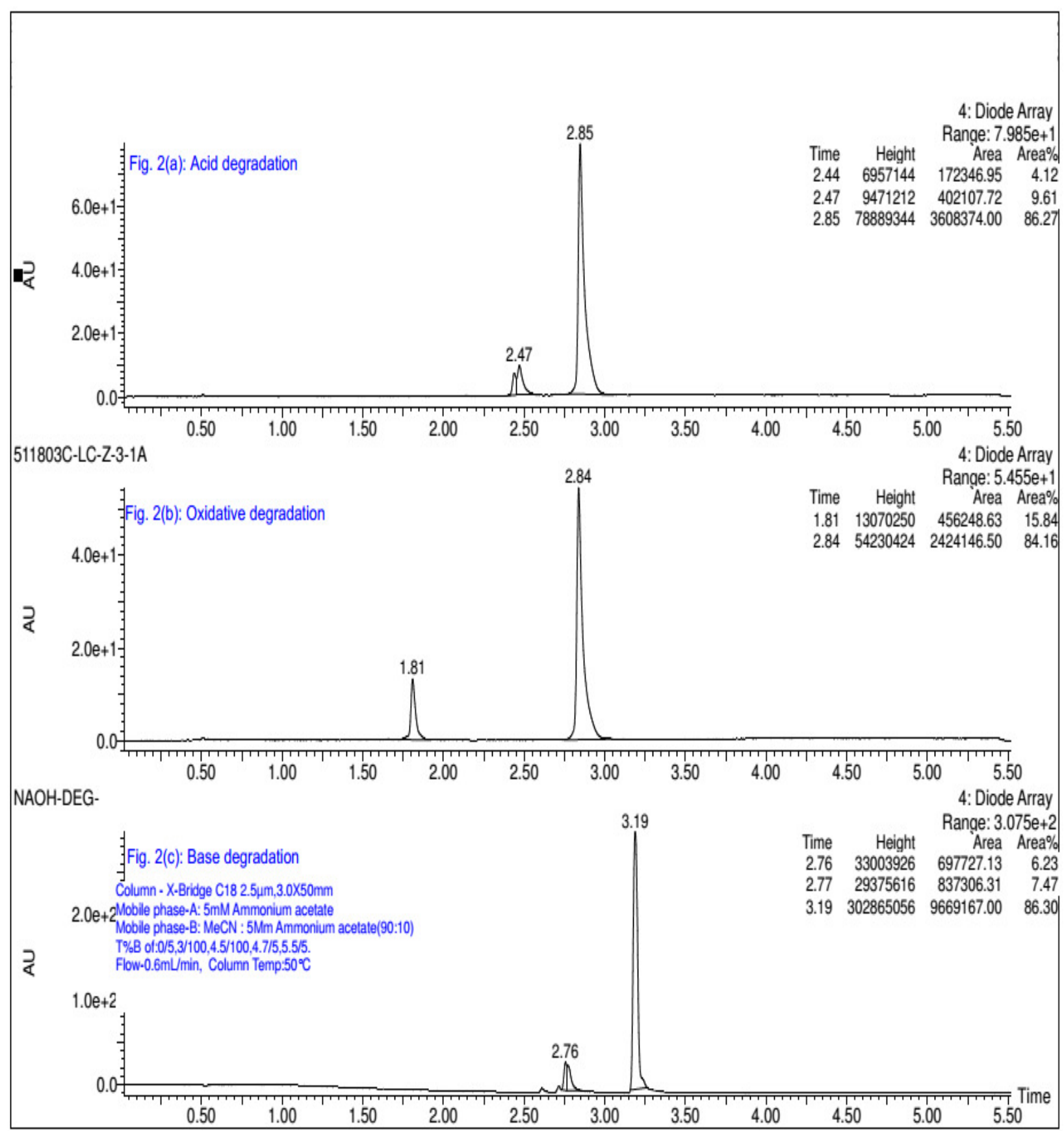

Fig.-2: Chromatograms of Acid, Peroxide and Base Mediated Degradation of Z-Isomer of Luliconazole

\section{High-Resolution Mass Spectrometry (HRMS)}

HRMS data were recorded with Q-Tof Micro Mass(from waters) and which is equipped with source ESI mode; and the MS parameters are: Capillary Voltage: 2800V; Sample Voltage:30V; Extraction cone voltage: $5 \mathrm{~V}$; Source Temp: $140^{\circ} \mathrm{C}$; Desolvation Temp: $300^{\circ} \mathrm{C}$; cone gas: $50 \mathrm{Lt} / \mathrm{hr}$; desolvation gas: 650Lt/hr. HRMS data for DP-1, DP-2, DP-4 \& DP-5 were analyzed with positive mode and DP-3 was recorded with Negative mode. Internal standard Leucine encephalin (m/z: 555.62268) was used for the calibration and as well as to check mass accuracy. MS data were recorded with Mass Lynx software. 


\section{Nuclear Magnetic Resonance Spectroscopy (NMR)}

The ${ }^{1} \mathrm{H},{ }^{13} \mathrm{C}$ and 2DNMR data of degradation impurities (DP-1 to DP-5) were recorded in deuterated dimethyl sulfoxide- $\mathrm{d}_{6}$ and methanol- $\mathrm{d}_{4}$ solvents on Bruker $400 \& 500 \mathrm{MHz}$ having consol Avance-IIIHD NMR instrument connected with Broad Band observe probe (BBO). Hydrogen and carbon chemical shifts are reported in parts per million, with respective to Tetramethylsilane as an internal standard. The spectra were set to chemical shift value $(\delta) 0.00 \mathrm{ppm}$ (Tetramethylsilane) in ${ }^{1} \mathrm{H}$ NMR and chemical shift value $(\delta)$ $39.50 \mathrm{ppm}$ in ${ }^{13} \mathrm{C}$ NMR for dimethyl sulfoxide- $\mathrm{d}_{6}$.

\section{Degradation Conditions}

Degradation studies are performed as per the ICH guidelines ${ }^{7}$. The drug substance was solubilized in $0.2 \mathrm{~N} \mathrm{HCl}$ and stirred for 10 hours at room temperature and the reaction was monitored by UP-LCMS. For oxidative stress condition, the drug substance was solubilized in $30 \% \mathrm{H}_{2} \mathrm{O}_{2}$ and stirred for 12 hours at room temperature and similarly, $0.2 \mathrm{~N} \mathrm{NaOH}$ is used for base stress conditions and stirred for 12hours at RT. Further $0.5 \mathrm{~mL}$ of acid, oxidative and base stress degradation solutions were solubilized in methanol and diluted with mobile phase and injected onto the UPLC-MS system. Thermal degradation was conducted by heating $50 \mathrm{mg}$ of drug substance at $80{ }^{\circ} \mathrm{C}$ for $12 \mathrm{hr}$, and $10 \mathrm{mg}$ of the heated drug was dissolved in 10mL of mobile phase (buffer \& acetonitrile 50:50), and further diluted with mobile phase to achieve 100 ppm solution. $3 \mu \mathrm{L}$ was injected on the UP-LCMS.

Photo stress condition was conducted by $50 \mathrm{mg}$ of drug was exposed to UV light in a UV chamber for 3days and $10 \mathrm{mg}$ of the UV irradiated drug was dissolved in $10 \mathrm{~mL}$ of mobile phase (buffer \& acetonitrile 50:50), and further diluted with mobile phase to achieve $100 \mathrm{ppm}$ solution. $3 \mu \mathrm{L}$ was injected on the UPLCMS.

\section{Experiment for Preparative HPLC}

Acid, oxidative and base degradation solutions were diluted with mobile phase (buffer \& acetonitrile 50:50), and injected in preparative HPLC one after other to isolate the new unknown degradation products DP-1 to DP-5. Hence, the isolated and collected preparative fractions were lyophilized to get solid material and these solid materials were further characterized.
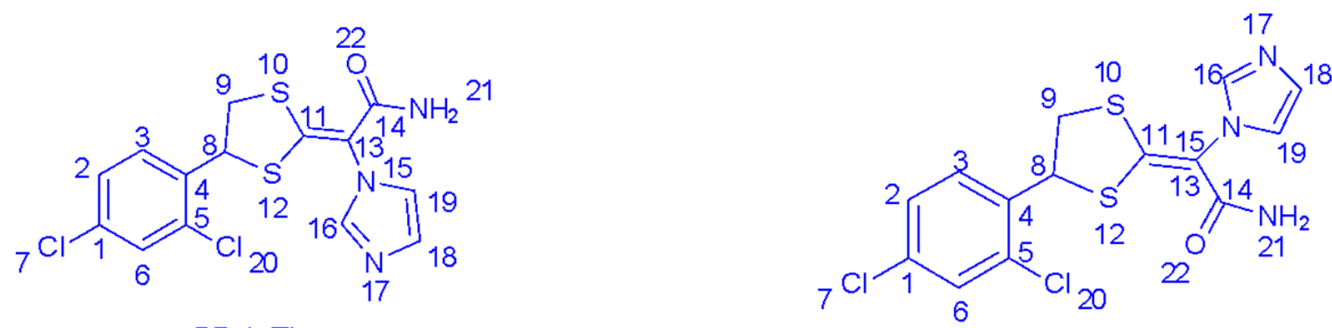

$\mathrm{DP}-1(\mathrm{Z})$
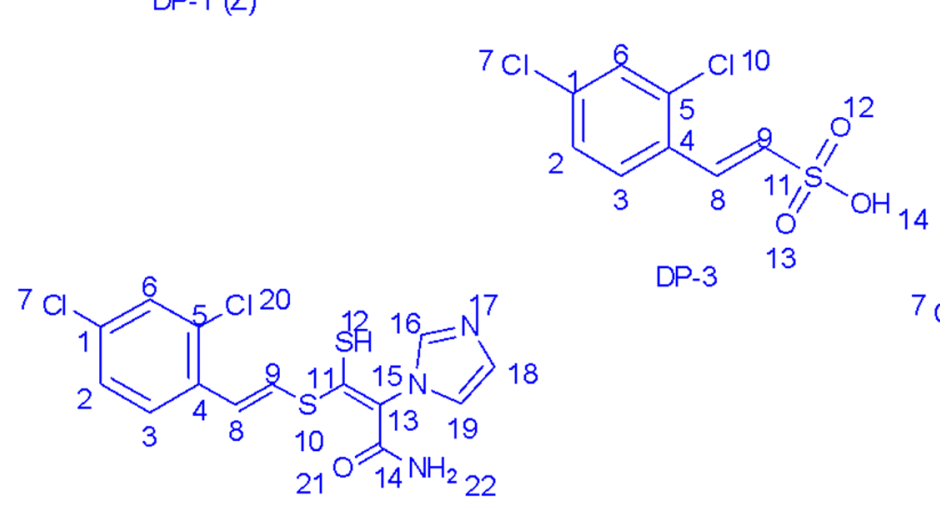

DP-2 (E)

DP-3

13

DP-4 (E)

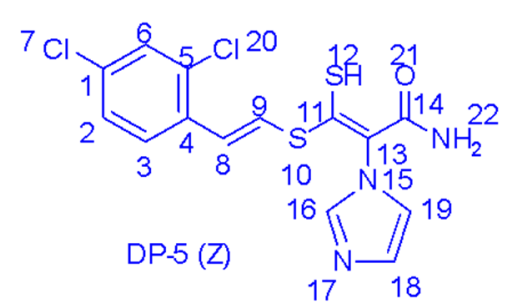

Fig.-3: Structures of Degradation Products (DP-1 to DP-5) 


\section{RESULTS AND DISCUSSION}

The acid treated Luliconazole Z-isomer (RZ) showed two close peaks (Fig.-2a, DP-1 (4.1\%), DP-2 (9.6\%), peroxide-treated Luliconazole Z-isomer (RZ) showed one peak (Fig.-2b, DP-3 (15.8\%)) and Luliconazole Z-isomer (RZ) treated with base showed two close peaks (Fig.-2c, DP-4 (6.2\%), DP-5 (7.4\%)). Luliconazole Z-isomer (RZ) was inert to heat and photo degradation, hence no degradation products were formed in heat and photo degradation study. Acid, oxidative and base treated solution was further incubated for $24 \mathrm{hr}$ to enhance the yield of degradation products and subjected to preparative HPLC for the isolation of peaks and structural characterization by 2DNMR and HRMS.

\section{Isolation of Acid, Oxidative and Base Degradation Products DP-1 to DP-5}

All the degradation products DP-1 to DP-5 were isolated by methods such as preparative HPLC and SFC described in the above sections. All five stress degradation products were structurally characterized by HRMS and NMR studies like nOe, 1D, and 2DNMR analysis. Fig. 3 shows the degradation structures of Z-Luliconazole.

\section{Structure elucidation of DP-1 and DP-2}

The ${ }^{1} \mathrm{H}$ NMR spectra of DP-1 and DP-2 when compared with drug substance showed two additional signals at $6.29 \mathrm{ppm}, 7.24 \mathrm{ppm}$ (for DP-1); 6.31 and 7.26 (for DP-2) integrating to one proton each. These two extra protons got exchanged in the D2O exchange experiment. The two additional signals which showed up in the degradants gave two cross peaks with a single nitrogen signal at $\sim 102 \mathrm{ppm}$ (both in DP-1 and DP-2) in ${ }^{15} \mathrm{~N}-\mathrm{HSQC}$ NMR data indicating that these two protons are attached to one nitrogen (-NH2). The ${ }^{1} \mathrm{H}$ NMR spectra of both DP-1 and DP-2 showed similarities with drug substance except for the two additional exchangeable protons. The HRMS of DP-1 is $\mathrm{m} / \mathrm{z} 371.9803$ and for DP-2 is $\mathrm{m} / \mathrm{z}$ 371.9800. This indicates that the cyano group got converted into an amide. And it was further confirmed by infra-red spectroscopy, $-\mathrm{CN}$ is observed at $2235.59 \mathrm{~cm}-1$ for drug substance and the same frequency was missing in DP-1 \& DP-2, and DP-1 and DP-2 were found to be geometrical isomers. In the Nuclear Overhauled Effect (nOe) study of DP-1, the signal at $7.17 \mathrm{ppm}(\mathrm{H}-18)$ was irradiated and the signals at 7.68, 7.66, 7.64 and $7.05 \mathrm{ppm}$ got enhanced corresponding to $\mathrm{H}-2, \mathrm{H}-3, \mathrm{H}-19$, and $\mathrm{H}-16$ protons indicating that the DP-1 is Z-isomer. In the nOe study of DP-2, H-18 was irradiated and none of the signals corresponding to H-2, H-3 got enhanced thus confirming that DP-2 as E-isomer (Fig.-3).

Table-1: ${ }^{1} \mathrm{H}$ and ${ }^{13} \mathrm{C}$ NMR Data of Z-Isomer of Luliconazole and Degradation Products DP-3, DP-4 and DP-5 in DMSO- $d_{6}$ on $400 \mathrm{MHz}$ at $25^{\circ} \mathrm{C}$; $*$ recorded in Methanol- $d_{4}$.

\begin{tabular}{|c|c|c|c|c|c|c|c|c|}
\hline $\begin{array}{c}\text { Assignm } \\
\text { ent }\end{array}$ & $\begin{array}{c}{ }^{1} \mathrm{H} \\
\text { Lulicona } \\
\text { zole-Z- } \\
\text { isomer }\end{array}$ & $\begin{array}{c}{ }^{13} \mathrm{C} \\
\text { Lulicon } \\
\text { azole- } \\
\mathrm{Z} \text { - } \\
\text { isomer }\end{array}$ & $\begin{array}{c}{ }^{1} \mathrm{H}^{*} \\
\mathrm{DP}-3\end{array}$ & $\begin{array}{l}{ }^{13} \mathrm{C}^{*} \\
\mathrm{DP}-3\end{array}$ & $\begin{array}{c}{ }^{1} \mathrm{H} \\
\mathrm{DP}-4(\mathrm{E})\end{array}$ & $\begin{array}{c}{ }^{13} \mathrm{C} \\
\mathrm{DP}-4(\mathrm{E})\end{array}$ & $\begin{array}{c}{ }^{1} \mathrm{H} \\
\mathrm{DP}-5(\mathrm{Z})\end{array}$ & $\begin{array}{c}{ }^{13} \mathrm{C} \\
\text { DP-5 (Z) }\end{array}$ \\
\hline 1 & - & 133.2 & - & 136.5 & - & 131.5 & - & 131.3 \\
\hline 2 & 7.51 & 127.6 & 7.35 & 128.9 & 7.34 & 127.1 & 7.40 & 127.7 \\
\hline 3 & 7.74 & 129.9 & 7.7 & 130.0 & 7.37 & 129.8 & 7.61 & 127.3 \\
\hline 4 & - & 133.5 & - & 132.9 & - & 134.2 & - & 133.9 \\
\hline 5 & - & 133.9 & - & 135.9 & - & 132.9 & - & 131.1 \\
\hline 6 & 7.72 & 129.2 & 7.51 & 130.7 & 7.56 & 128.9 & 7.55 & 128.9 \\
\hline 7 & - & - & - & - & - & - & - & - \\
\hline 8 & 5.70 & 54.5 & 7.61 & 130.4 & 6.58 & 116.7 & 6.62 & 117.3 \\
\hline 9 & $\begin{array}{c}4.25 \& 4.1 \\
1 \\
\end{array}$ & 43.7 & 7.02 & 135.0 & 8.03 & 132.1 & 8.26 & 133.2 \\
\hline 10 & - & - & - & - & - & - & - & - \\
\hline 11 & - & 166.9 & - & - & - & 167.2 & - & 167.6 \\
\hline 12 & - & - & - & - & - & - & - & - \\
\hline 13 & - & 92.1 & - & - & - & 119.4 & - & 119.1 \\
\hline 14 & - & 115.3 & - & - & - & 166.3 & - & 166.1 \\
\hline
\end{tabular}


RASĀYAN J. Chem.

Vol. 12 | No. 2 |691 - 696| April - June | 2019

\begin{tabular}{c|c|c|l|c|c|c|c|c}
\hline 15 & - & - & & & - & - & - & - \\
\hline 16 & 7.93 & 137.6 & & & 7.22 & 139.6 & 7.22 & 139.5 \\
\hline 17 & - & - & & & - & - & - & - \\
\hline 18 & 7.42 & 119.9 & & & 6.84 & 126.6 & 6.84 & 126.6 \\
\hline 19 & 7.09 & 129.6 & & & 6.77 & 122.8 & 6.78 & 122.5 \\
\hline 20 & - & - & & & - & - & - & - \\
\hline 21 & - & - & & & - & - & - & - \\
\hline 22 & & & & & $6.84,10.3$ & - & $6.81,10.2$ & - \\
\hline
\end{tabular}

\section{Structure Elucidation of DP-3}

The HRMS of DP-3 found a base peak at 250.9340 corresponding to the molecular formula $\mathrm{C}_{8} \mathrm{H}_{5} \mathrm{O}_{3} \mathrm{SCl}_{2}$ $[\mathrm{M}-\mathrm{H}]$ in negative mode. The ${ }^{1} \mathrm{H}$ NMR spectrum in DMSO-d6 showed around nine protons only in the aromatic region. However, the D2O exchange experiment showed only five protons. It is possible that some protons are due to salt formation. Further1D and 2DNMR data confirms that the compound to be (E)-2-(2,4-dichlorophenyl) ethane sulfonic acid (Fig. 3). The protons $\mathrm{H}-8$ and $\mathrm{H}-9$ are Trans to each other by coupling constant $15-16 \mathrm{~Hz} .{ }^{1} \mathrm{H}$ and ${ }^{13} \mathrm{CNMR}$ assignments are given in Table-1.

\section{Structure Elucidation of DP-4 and DP-5}

The HRMS of DP-4 and DP-5 showed base peaks at m/z at 371.9802 and m/z 371.9810 respectively. Although DP-1, DP-2 and DP-3, DP-4 showed similar molecular masses, there are changes in their ${ }^{1} \mathrm{H}$ NMR spectra. The ${ }^{1}$ HNMR spectra of both DP-4 and DP-5 shows similar patterns with no aliphatic protons. Whereas three aliphatic protons were observed in ${ }^{1} \mathrm{HNMR}$ spectra of DP-1 and DP-2., Analysis of HSQC, HMBC NMR data of DP-4 and DP-5 indicated that the opening of the 1,3dithiolane ring resulting in thiol formation. DP-4 and DP-5 were found to be geometrical isomers. In the nOe study of DP-4, the signal at H-8 (6.58ppm) was irradiated and the signals corresponding to the imidazole ring did not get enhanced, indicating that the DP-4 is E-isomer. In the nOe study of DP-5, H-8 (6.64ppm) was irradiated and H-16 (7.22 ppm) got enhanced thus confirming that DP-5 is Z-isomer. The structures of DP-4 and DP-5 are shown in Fig. 3 and their NMR spectral data is shown in Table-1.

\section{CONCLUSION}

A total of five novel Luliconazole Z-Isomer stress degradation products were found in the acid, base and oxidative degradation study. All the five degradation products were structurally characterized by IR, HRMS, and NMR studies. The geometrical isomers in the degradants have been characterized using 1D nOe. Further studies are required to characterize the formation of Luliconazole Z-Isomer degradants during storage of Luliconazole E-Isomer and their safety evaluation.

\section{ACKNOWLEDGMENT}

The authors wish to thank the management of GVK Biosciences Pvt. Ltd. for supporting this work.

\section{REFERENCES}

1. K. Hiroki, N. Yoshimi,K. Kazuo, Y. Masanori, WO002821A2 (1997)

2. Y. Niwano, T. Ohm, A. Seo, H. Kodama, H. Koga, A. Sakai, Curr. Med. Chem., 2, 147(2003)

3. Y. Niwano, N. Kuzuhara, H. Kodama, M. Yoshida, T. Miyazaki, H. Yamaguchi, Agents.Chem. 42, 967(1998)

4. Y. Niwano, N. Kuzuhara, Y. Goto, Y. Munechika, H. Kodama, K. Kanai, M. Yoshida, T. Miyazaki, H. Yamaguchi, Int. J. Antimicrob. Agents, 12, 221(1999)

5. S. S. Sona wane, G. Parag, Arabian J. Chem., 9, S1428(2016), DOI: 10.1016/j.arabjc.2012.03.019

6. M. AditiMalasiya, G. Anju, Int. J. Pharm. Chem. Anal, 4, 46(2017)

7. ICH guideline (2003) Q1A (R2) Stability Testing of New Drug Substances and Products. In: International Conference on Harmonization, IFPMA, Geneva.

[RJC-5087/2018] 